\title{
Diurnal Variation in P-glycoprotein-Mediated Transport and Cerebrospinal Fluid Turnover in the Brain
}

\author{
Laura Kervezee, ${ }^{1,2}$ Robin Hartman, ${ }^{1}$ Dirk-Jan van den Berg, ${ }^{1}$ Shinji Shimizu, ${ }^{1}$ Yumi Emoto-Yamamoto, ${ }^{1}$ \\ Johanna H. Meijer, ${ }^{2,3}$ and Elizabeth C. M. de Lange ${ }^{1,3}$
}

Received 15 April 2014; accepted 19 May 2014; published online 11 June 2014

\begin{abstract}
Nearly all bodily processes exhibit circadian rhythmicity. As a consequence, the pharmacokinetic and pharmacodynamic properties of a drug may also vary with time of day. The objective of this study was to investigate diurnal variation in processes that regulate drug concentrations in the brain, focusing on P-glycoprotein (P-gp). This efflux transporter limits the distribution of many drugs in the brain. To this end, the exposure to the P-gp substrate quinidine was determined in the plasma and brain tissue after intravenous administration in rats at six different time points over the 24 -h period. Our results indicate that time of administration significantly affects the exposure to quinidine in the brain. Upon inhibition of P-gp, exposure to quinidine in brain tissue is constant over the 24-h period. To gain more insight into processes regulating brain concentrations, we used intracerebral microdialysis to determine the concentration of quinidine in brain extracellular fluid (ECF) and cerebrospinal fluid (CSF) after intravenous administration at two different time points. The data were analyzed by physiologically based pharmacokinetic modeling using NONMEM. The model shows that the variation is due to higher activity of P-gp-mediated transport from the deep brain compartment to the plasma compartment during the active period. Furthermore, the analysis reveals that CSF flux is higher in the resting period compared to the active period. In conclusion, we show that the exposure to a P-gp substrate in the brain depends on time of administration, thereby providing a new strategy for drug targeting to the brain.
\end{abstract}

KEY WORDS: blood-brain barrier; chronobiology; intrabrain distribution; microdialysis; PBPK modeling.

\section{INTRODUCTION}

The treatment of brain tumors, infectious diseases, and various neurological disorders is often unsuccessful because the entry of most clinically available drugs into the brain is restricted (1). This is due to a number of protective mechanisms that prevent the distribution of potentially toxic compounds in the brain but, at the same time, impede drugs from reaching their target site in the central nervous system (CNS). For example, several types of efflux transporters are expressed in the brain, which expel a wide variety of exogenous substances from the brain back into the circulation $(1,2)$.

Johanna H. Meijer and Elizabeth C.M. de Lange contributed equally to this work.

Electronic supplementary material The online version of this article (doi:10.1208/s12248-014-9625-4) contains supplementary material, which is available to authorized users.

$\overline{{ }^{1} \text { Division of Pharmacology, LACDR, Leiden University, PO Box }}$ 95022300 RA, Leiden, The Netherlands.

${ }^{2}$ Laboratory for Neurophysiology, Department of Molecular Cell Biology, Leiden University Medical Center, PO Box 9600 Mailbox S5-P2300 RC, Leiden, The Netherlands.

${ }^{3}$ To whom correspondence should be addressed. (e-mail: J.H.Meijer@lumc.nl; ecmdelange@lacdr.leidenuniv.nl)
A well-known efflux transporter in the CNS is Pglycoprotein (P-gp). At the level of the blood-brain barrier (BBB), it restricts the entry of many, mainly hydrophobic, drugs to the brain (3). Although P-gp is mostly known for its role in the efflux of its substrates at the BBB, it is also expressed in blood-cerebrospinal fluid (CSF) barrier (BCSFB) as well as in parenchymal cells types, such as astrocytes and pericytes (4). Several strategies have been proposed to diminish P-gp-mediated efflux, but it has been difficult to implement these strategies in the clinic and, especially, to apply them to chronic diseases (5).

The pharmacokinetic and pharmacodynamic properties of a wide variety of drugs show diurnal variation, which is a result of 24-h rhythms in, for example, gastrointestinal function, activity of xenobiotic-metabolizing enzymes, blood flow, and glomerular filtration rate (6). Consequently, therapeutic efficiency as well as the severity of side effects of drugs may vary with time of day (7-9). Regarding P-gp, it has been shown that its expression and activity show a diurnal rhythm in the intestines of rodents, leading to different plasma concentrations of orally administered P-gp substrates depending on the time of administration (10-15).

The objective of this study was to examine whether the distribution of P-gp substrates in the CNS depends on time of administration. Diurnal variation in P-gp activity could be exploited to either increase or reduce the effect of P-gp on 
CNS target site distribution, depending on whether the aim is to limit or enhance CNS distribution of the drug. Other processes that govern CNS target site concentrations (16), such as CSF turnover, may also vary over the course of the day. Understanding the effect of time of administration on CNS target site distribution may be useful to improve the efficiency of therapies involving P-gp substrates.

In this study, the concentration of the P-gp substrate quinidine was determined in plasma and brain tissue after intravenous administration at six different time points over the 24-h period in rats. As we observed a significant 24-h variation in P-gp-mediated drug transport in brain tissue, we next used intracerebral microdialysis to obtain quinidine concentration-time profiles in brain extracellular fluid (ECF) and in CSF at two different times of the day. Using physiologically based pharmacokinetic (PBPK) modeling to describe quinidine brain distribution (17), we were able to explore differences in P-gp-mediated transport and CSF flux between the active and resting period of the animals. The results show that time is an important consideration in the design of drug treatments targeted at the CNS.

\section{MATERIALS AND METHODS}

\section{Animals}

Male Wistar rats (Charles River, The Netherlands) were housed in groups under standard environmental conditions (12:12LD cycle) with free access to water and food (SDS, Technilab-BMI, Someren, the Netherlands). After surgery, the rats were kept individually. All animal procedures were performed in accordance with the Dutch law on animal experimentation and were approved by the Animal Ethics Committee of the Leiden University (protocol number DEC12088).

\section{Chemicals and Solutions}

Quinidine, quinidine sulfate dehydrate, and quinine were obtained from Sigma-Aldrich (Zwijndrecht, the Netherlands); tariquidar from Xenova Group PLC (Cambridge, UK); saline and $5 \%$ glucose in saline from the Leiden University Medical Centre (Leiden, the Netherlands); Heparin from LEO Pharma BV (Breda, the Netherlands); nembutal and isoflurane from AUV (Cuijk, the Netherlands); phosphoric acid and boric acid from Merck (Darmstadt, Germany), sodium hydroxide and triethyl amine (TEA) from Baker (Enschede, the Netherlands); and methanol, methyl tert-butyl ether (TBME), and acetonitrile from Biosolve (Valkenswaard, the Netherlands). Perfusion fluid (PF) for microdialysis experiments was prepared as described in (18).

\section{Surgery}

Animals were anesthetized by isoflurane during surgical procedures (induction, 5\%; maintenance, 1-2\%). A cannula was inserted in the femoral artery and femoral vein 7 days prior to the experiment as described previously (17). Animals used for microdialysis experiments also received microdialysis guides with dummy probes (CMA, Solna, Sweden) in the caudate putamen $(\mathrm{CP}$; coordinates from bregma, AP-
$1.0 \mathrm{~mm} \mathrm{~L}+3.0 \mathrm{~mm} \mathrm{~V}-3.4 \mathrm{~mm}$ ) and in the cisterna magna (CM; coordinates from lambda, AP-2.51 mm L+2.04 mm V$8.34 \mathrm{~mm}$, at an angle of $25^{\circ}$ anterior from the dorsoventral axis and $11^{\circ}$ lateral from the anteroposterior axis) (17). Twenty-four hours before the experiment, the dummy probes in $\mathrm{CP}$ and $\mathrm{CM}$ were replaced by 4 and $1 \mathrm{~mm}$ microdialysis probes (CMA), respectively.

\section{Drug Administration, Serial Blood Sampling, and Collection of Brain Tissue}

Brain distribution experiments were performed at six different time points across the 24-h period; $t=0$ (start of quinidine administration) was at Zeitgeber Time (ZT) 0, ZT4, ZT8, ZT12, ZT16, or ZT20 ( \pm 10 min), with ZT12 defined as the moment of lights off. Microdialysis and in vivo recovery experiments were performed with $t=0$ at ZT8 or ZT20. The number of animals per group is shown in Supplemental Table I. Between ZT12 and ZT0 experiments were conducted in dim red light.

Animals were pretreated with an intravenous infusion of vehicle (5\% glucose in saline) or $15 \mathrm{mg} / \mathrm{kg}$ tariquidar in $5 \%$ glucose at $t=-25 \mathrm{~min}$ for $10 \mathrm{~min}$ at a rate of $500 \mu \mathrm{L} / \mathrm{min} / \mathrm{kg}$ with a syringe pump (Pump 22 Multiple Syringe Pump, Harvard Apparatus, Holliston, MA, USA). In brain distribution and microdialysis experiments, quinidine $(10 \mathrm{mg} / \mathrm{kg})$ was administered intravenously at $t=0$ in $10 \mathrm{~min}$ at a rate of $250 \mu \mathrm{L} / \mathrm{min} / \mathrm{kg}$. Blood samples $(100 \mu \mathrm{L})$ were collected at $t=$ $-10,10,30,60,90,120,150,180,210$, and $240 \mathrm{~min}$ in heparinized Eppendorf tubes and centrifuged for $10 \mathrm{~min}$ at 5,000 rpm (Eppendorf Microcentrifuge Model 5415D) to obtain plasma. Plasma was stored at $-20^{\circ} \mathrm{C}$ until analysis. At $t=240 \mathrm{~min}$, the rats were sacrificed with an intravenous injection of Nembutal and transcardially perfused with PBS until the organs were free of blood. Brain tissue was removed and stored at $-80^{\circ} \mathrm{C}$.

\section{Intracerebral Microdialysis}

Microdialysis probe inlets were connected to a syringe filled with $\mathrm{PF}$, and the outlets were connected to a fraction collector (Univentor microsampler 820, Univentor Ltd, Zejtun, Malta) with FEP tubing. Perfusion of the microdialysis probes with a syringe pump (Bioanalytical Systems Inc., West Lafayette, USA) started $2 \mathrm{~h}$ prior to quinidine administration. Perfusion rate was set to $1 \mu \mathrm{L} / \mathrm{min}$. Samples were collected every $20 \mathrm{~min}$ until $t=240 \mathrm{~min}$ and stored at $-80^{\circ} \mathrm{C}$.

\section{In Vivo Retrodialysis}

The recovery of quinidine into the microdialysis probe was determined by in vivo retrodialysis. Probes were perfused with blank PF for 2 hours, after which the concentration of quinidine in PF was changed every $2 \mathrm{~h}$ from 20 to $50 \mathrm{ng} / \mathrm{mL}$ and to $200 \mathrm{ng} / \mathrm{mL}$. Samples were collected every $20 \mathrm{~min}$ at a perfusion rate of $1 \mu \mathrm{L} / \mathrm{min}$. The probe recovery (extraction fraction) was calculated as described by Westerhout et al. (2013). The extraction fractions from the probes located in $\mathrm{CP}$ and $\mathrm{CM}$ were used to convert the measured 
microdialysate concentrations to ECF and CSF concentrations, respectively.

\section{Plasma Protein Binding}

To determine the degree of plasma protein binding of quinidine, $50 \mu \mathrm{L}$ aliquots of each plasma sample taken at $t=$ $30 \mathrm{~min}$ and $t=90 \mathrm{~min}$ were pooled per experimental time point (ZT) and pre-treatment (vehicle or TQD). The samples were equilibrated at $37^{\circ} \mathrm{C}$ for $30 \mathrm{~min}$, and $200 \mu \mathrm{L}$ was added to a pre-heated Centrifree $30 \mathrm{~K}$ ultrafiltration device (Millipore BV, Etten-Leur, the Netherlands). The samples were centrifuged for $20 \mathrm{~min}$ at $5,000 \mathrm{rpm}$ at $37^{\circ} \mathrm{C}$ according to the manufacturer's protocol. The unbound fraction of quinidine in plasma ( $f_{\text {unbound }}$ ) was calculated by dividing the concentration of the ultrafiltrate by the concentration in the unfiltered sample.

\section{Measurement of Quinidine Concentration in Microdialysate, Plasma, and Brain Homogenates}

Quinidine concentrations in plasma, microdialysates, and brain homogenates were measured using high-performance liquid chromatography (HPLC) with fluorescence detection as described earlier (19). Microdialysates $(10 \mu \mathrm{L})$ were directly injected into the HPLC system using a mobile phase with an acetonitrile/buffer ratio of 17:83 (v/v). Brain tissue was homogenized in $50-\mathrm{mM}$ phosphate buffer $(\mathrm{pH} 7.4)$ using the Bullet Blender 5 (Next Advance Inc., NY, USA) according to the manufacturer's protocol. Brain homogenates were diluted $6 \times(w / v)$ in $50-\mathrm{mM}$ phosphate buffer $(\mathrm{pH} 7.4)$. Extraction of quinidine from plasma and brain homogenate was performed as described earlier (19). The injection volume was $2-20 \mu \mathrm{L}$ depending on the type of sample. The mobile phase consisted of acetonitrile and buffer in a ratio of $14: 86(v / v)$.

\section{Data Analysis}

Areas under the curve (AUC in $\mathrm{ng} / \mathrm{mL} \times \min$ ) of unbound quinidine in plasma from 0 to $240 \mathrm{~min}$ after administration $\left(\mathrm{AUC}_{\mathrm{PLu}, 0-240}\right)$ were calculated using the trapezoidal rule. Ratios between parameters were determined for each animal individually before calculating the mean per group. The means of two groups were compared by an unpaired Student's $t$ test (normally distributed data) or a Mann-Whitney $U$ test (not normally distributed data). To compare the means of more than two groups, an ANOVA (normally distributed data) or Kruskal-Wallis test (not normally distributed data) was used. Normal distribution was assessed by the Sharipo-Wilk test. $p$ values below 0.05 were considered significant. Statistical analyses and graphical visualization were performed with $\mathrm{R}$ version 2.14.1 or version 3.0.1 (R Foundation for Statistical Computing, Vienna, Austria).

\section{Physiologically Based Pharmacokinetic Modeling}

PBPK brain distribution modeling was performed to investigate the effect of time of administration on the pharmacokinetics of quinidine in plasma, total brain, ECF, and CSF quantitatively using a nonlinear mixed effect model approach with NONMEM software version VII (GloboMax LLC, Hanover, MD, USA). The PBPK model describing brain distribution of quinidine that was previously published (17) was applied to the data (Supplemental Figure 1). Data obtained during the microdialysis experiments in this study were added to the previously obtained data produced with the same experimental method during the resting period of the animals (17). Using covariate analysis, the effect of time of administration was tested on parameters describing P-gpmediated transport and CSF flux. Statistical significance was based on changes in the objective function value (OFV; $p<$ $0.05)$. The difference in the OFV obtained by comparing each model was assumed to be asymptotically chi-squared distributed with degrees of freedom (df) equal to the difference in the number of parameters between the two models. Goodness-of-fit plots (model predicted $v s$. observed data values) were evaluated by diagnostic scatter plots. The stability and performance of the final model were confirmed by a visual predictive check on the simulated distribution of the concentration data to cover over $90 \%$ of the observed data.

\section{RESULTS}

\section{Time of Administration Has No Effect on Plasma Pharmacokinetics of Quinidine}

We first determined the mean concentration-time profiles of unbound quinidine in plasma after intravenous administration at six experimental time points (ZT0-20) (Fig. 1a). Unbound plasma concentrations were derived from total plasma concentrations corrected for by the unbound fraction in plasma $(0.286 \pm 0.006)$. Total and unbound concentrations of quinidine in plasma showed a highly significant linear correlation $\left(r^{2}=0.953, N=24, p=0.000\right.$, Pearson's correlation; Supplemental Figure 2A). The unbound fraction did not depend on time of administration $(\mathrm{F}(5,17)=2.52, p=$ 0.07, two-way ANOVA; Supplemental Figure 2B) and pretreatment with TQD $(\mathrm{F}(1,17)=1.70, p=0.21$, two-way ANOVA; Supplemental Figure 2C)

The unbound quinidine concentration in plasma in vehicle-treated animals at $t=30,90,120,150,210$, and 240 was influenced by time of administration $(p<0.05$, one-way ANOVA or Kruskal-Wallis, as appropriate; Fig. 1a). Time of administration had no significant effect on the area under the time concentration curve of unbound quinidine in plasma from 0 to $240 \mathrm{~min}$ after administration $\left(\mathrm{AUC}_{\mathrm{PLu}(0-240)}\right)$ (Fig. 1b $\mathrm{F}(5,34)=1.49, p=0.220$, one-way ANOVA). These results indicate that the total exposure to quinidine in plasma is not affected by time of administration.

\section{Quinidine Brain Tissue Concentration Shows Significant Diurnal Variation}

The concentration of quinidine in brain tissue at $t=240$ $\left(C_{B R}\right)$ relative to $A U C_{P L u(0-240)}$ was found to be significantly affected by time of administration (Fig. 1c $\mathrm{H}(5)=14.9$, $p=$ 0.011 , Kruskal-Wallis test). In experiments conducted in the resting period of the animals (i.e., animals treated at ZT0, $\mathrm{ZT} 4$, and $\mathrm{ZT} 8)$, the $\mathrm{C}_{\mathrm{BR}}$ : $\mathrm{AUC}_{\mathrm{PLu}(0-240)}$ was on average twice as high compared to the ratio in the active period (at ZT12, 

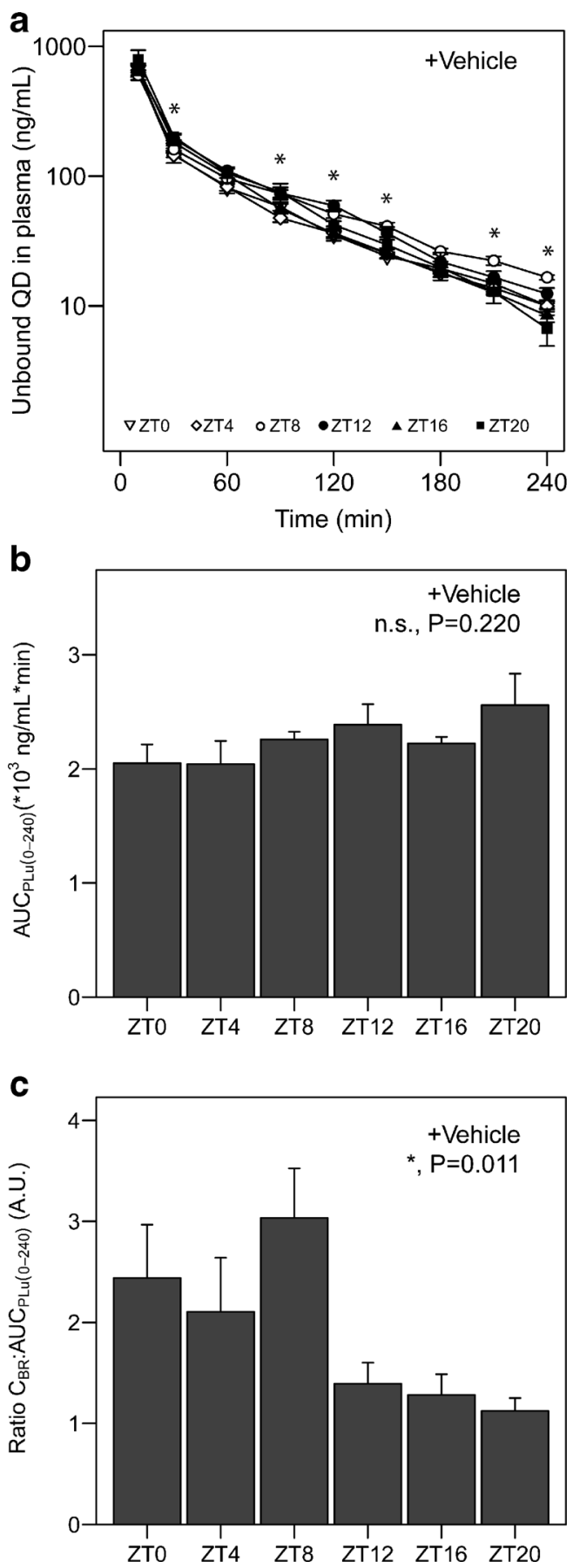

Fig. 1. Quinidine exposure in plasma and brain in vehicle-treated animals. a Time-concentration profiles of unbound quinidine in plasma. Different symbols represent the different experimental time points (ZT0, 4, 8, 12, 16, and 20). Asterisks indicate significant effect of ZT on plasma concentration at the designated sampling times ( $n=6-8$ animals per group). b Area under the curve for unbound quinidine in plasma from 0 to $240 \mathrm{~min}$ after administration (AUC $\mathrm{PLu}, 0-240)$ at different experimental time points ( $n=5-8$ animals per group). No significant time of day effect on $\mathrm{AUC}_{\mathrm{PLu}, 0-240}$ was found ( $p>0.05$, one-way ANOVA). c Quinidine concentration at $t=240 \mathrm{~min}$ in brain homogenate relative to plasma AUC $\left(\mathrm{C}_{\mathrm{BR}}\right.$ : $\left.\mathrm{AUC}_{\mathrm{PLu}, 0-240}\right)$ at different experimental time points ( $n=4-8$ animals per group). Time of administration significantly affects this ratio $(p<0.05$, Kruskal-Wallis rank sum test). Mean \pm SEM in all graphs. n.s. not significant; *p $<0.05$
ZT16, or ZT20). These results indicate that the exposure to the P-gp substrate quinidine in the brain, relative to plasma exposure, exhibits diurnal variation.

\section{Diurnal Variation of Total Brain Quinidine Concentrations is Due to the Variation in P-gp-Mediated Transport}

To determine whether the effect of time of administration on the exposure to quinidine in brain tissue is due to variation in P-gp-mediated transport over the 24-h period, we administered the selective $\mathrm{P}$-gp inhibitor tariquidar intravenously $30 \mathrm{~min}$ prior to quinidine administration at ZT0, $4,8,12,16$, or 20 . Time of administration did not significantly affect unbound plasma concentrations of quinidine at any of the sampling points (Fig. 2a) and had no effect on $\mathrm{AUC}_{\mathrm{PLu}(0-240)}$ (Fig. 2b F(5,33)= $0.635, p=0.675$, one-way ANOVA) in tariquidar-treated animals, indicating that also in this group of animals, time of administration does not affect exposure to quinidine in plasma. Importantly, time of administration also did not significantly affect $\mathrm{C}_{\mathrm{BR}}$ : $\mathrm{AUC}_{\mathrm{PLu}(0-240)}$ of quinidine after tariquidar treatment (Fig. 2c $\mathrm{F}(5,31)=1.28, p=0.297$, one-way ANOVA). Hence, tariquidar pre-treatment abolished the diurnal variation in $\mathrm{C}_{\mathrm{BR}}: \mathrm{AUC}_{\mathrm{PLu}(0-240)}$ that was observed in vehicle-treated animals. These results indicate that the exposure to quinidine in the brain relative to plasma levels exhibits diurnal variation unless P-gp is inhibited.

\section{Exposure to Quinidine in CNS is Influenced by Diurnal Variation in P-gp Activity and CSF Flux}

Next, we used intracerebral microdialysis to determine unbound concentrations of quinidine in brain extracellular fluid (ECF) and cerebrospinal fluid (CSF) from 0 to $240 \mathrm{~min}$ after intravenous quinidine administration at ZT8 (resting period) and ZT20 (active period of the animals). The extraction fractions ( $4 \mathrm{~mm}$ probe, $13 \pm 1.4 \% ; 1 \mathrm{~mm}$ probe, $8.4 \pm 2.6 \%$ ) were used to calculate unbound concentrations in ECF and CSF from microdialysate concentrations.

We calculated the mean concentration-time profiles of unbound quinidine in plasma, ECF, and CSF (Fig. 3) as well as $\mathrm{C}_{\mathrm{BR}}$ : $\mathrm{AUC}_{\mathrm{PLu}(0-240)}$ (Supplemental Figure 3B-C) at ZT8 and ZT20 in vehicle- and TQD-treated animals. The fraction unbound of quinidine in plasma $(0.276 \pm 0.015)$ was not significantly different in these experiments compared to that observed in the brain distribution experiments $(\mathrm{t}(29)=0.747, p=0.461$, two-sided $t$ test, Supplemental Figure 2D). Also, AUC $\mathrm{PLu}_{\mathrm{PL}(240)}$ and $\mathrm{C}_{\mathrm{BR}}$ : $\mathrm{AUC}_{\mathrm{PLu}(0-240)}$ measured in these experiments were comparable to those obtained in brain distribution experiments (Supplemental Figure 3). In vehicle-treated animals, ECF concentrations were not significantly affected by time of administration at any of the sampling times (Fig. 3c), while CSF concentrations were higher in the first $100 \mathrm{~min}$ after administration in the active period compared to the values obtained after administration in the resting period (Fig. 3e). In tariquidar-treated animals, time of administration significantly affected ECF concentrations at two sampling times (Fig. 3d), but had no effect on CSF concentrations (Fig. 3f).

The data from the microdialysis experiments were fitted to a PBPK model that was previously used to describe quinidine pharmacokinetics in plasma, ECF, CSF, and deep brain tissue (17). We investigated the effect of time of 
a

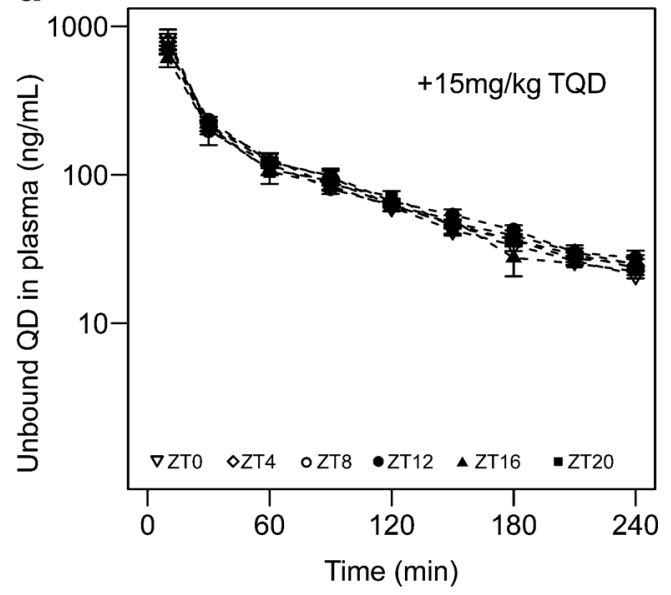

b

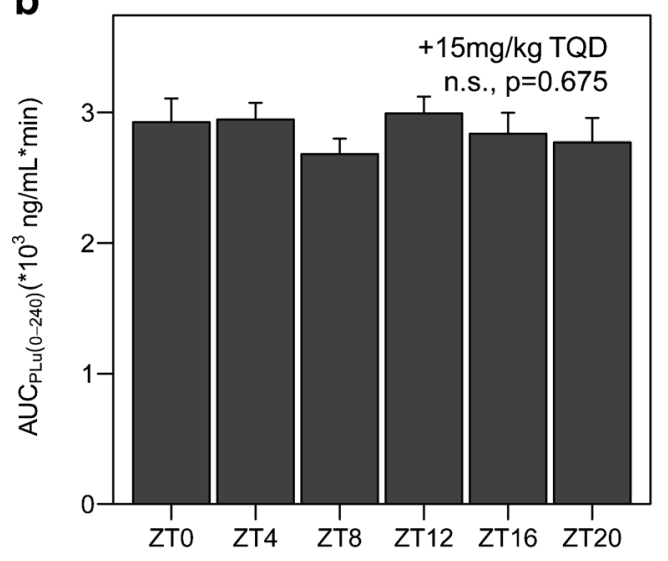

C

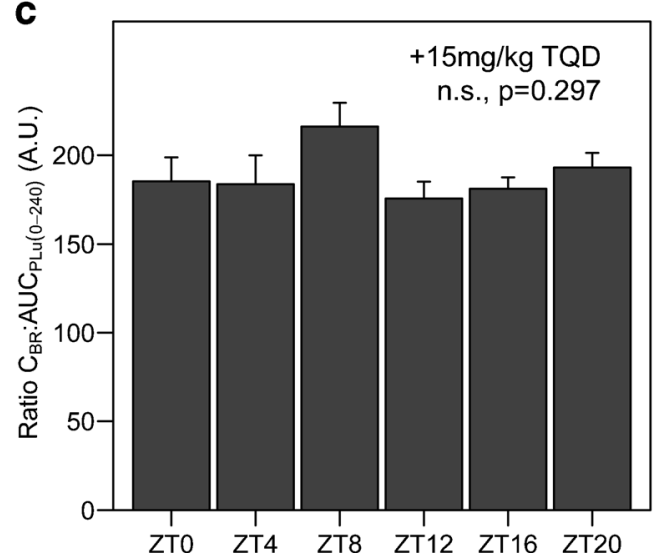

Fig. 2. Quinidine exposure in plasma and brain in tariquidar-treated animals. a Time-concentration profiles of unbound quinidine in plasma. Different symbols represent the different experimental time points (ZT0, 4, 8, 12, 16, or 20). No significant effect of ZT was found between plasma concentrations at different sampling times $(n=6-8$ animals per group). b Area under the curve for unbound quinidine in plasma from 0 to $240 \mathrm{~min}$ after administration $\left(\mathrm{AUC}_{\mathrm{PLu}, 0-240}\right)$ at different experimental time points $(n=5-8$ animals per group). No significant time of day effect on $\mathrm{AUC}_{\mathrm{PLu}, 0-240}$ was found $(p>0.05$, one-way ANOVA). c Quinidine concentration at $t=240 \mathrm{~min}$ in brain homogenate relative to plasma $\mathrm{AUC}\left(\mathrm{C}_{\mathrm{BR}}\right.$ : $\left.\mathrm{AUC}_{\mathrm{PLu}, 0-240}\right)$ at different experimental time points ( $n=4-8$ animals per group). No significant time of day effect was found on this ratio in tariquidar-treated animals ( $p>0.05$, one-way ANOVA). Mean \pm SEM in all graphs. $n . s$. not significant administration on the parameters describing P-gp-mediated transport from the plasma compartment to and from deep brain tissue, ECF and CSF, and on CSF flux. Using covariate analysis, we found that the model that takes into account the effect of time of administration on both P-gp-mediated transport and CSF flux best described the data (Table I), indicating that time of administration significantly influences P-gp-mediated transport and CSF flux. The parameter estimates of the model (Table II) show that the estimated Pgp-mediated transport of quinidine from the deep brain compartment to plasma is almost $2.9 \times$ higher during the active period of the animals compared to the resting period (659 vs $228 \mu \mathrm{L} / \mathrm{min}$, Table II). Furthermore, CSF flux is almost twice as high during the resting period of the animals compared to the active period $(0.522$ vs $0.227 \mu \mathrm{L} / \mathrm{min}$, Table II).

\section{DISCUSSION}

In this study, we show that the exposure to the P-gp substrate quinidine in total brain tissue is subject to significant diurnal variation. This variation is abolished by pre-treatment with the selective P-gp inhibitor tariquidar, indicating a selective P-gp-mediated origin. The exposure to quinidine in brain tissue is lower during the active period compared to the resting period of the animals, which shows that the activity of $\mathrm{P}$-gp is elevated during the active period. PBPK modeling based on plasma, ECF, CSF, and total brain concentrations of quinidine after intravenous administration in the active and resting period of the animals supports these findings by showing that time of administration significantly affects P-gpmediated transport as well as CSF flux. Therefore, the findings presented in this study show that the exposure to a drug in the brain is affected by time of administration and provides a mechanism that involves $24-\mathrm{h}$ variation in the activity of P-gp-mediated transport and CSF flux.

Circadian rhythms are present in many bodily processes and are controlled by a central clock located in the hypothalamus (20). Rhythms in peripheral organs are synchronized by the central clock, but are driven locally by an intracellular translational-transcriptional feedback loop with a period of approximately 24-h (21). This clock mechanism is responsible for the circadian rhythm that is found in the transcription, translation, and post-translational modification of many genes and their associated proteins (21). Our results show that the distribution of quinidine in the brain depends on time of administration due to variation in two processes. Firstly, we find that the estimated activity of P-gp-mediated transport is higher during the active period of the animals compared to the resting period. This is in line with several in vitro and in vivo studies that have shown 24-h variation in the expression and activity of P-gp in several different cell types (10-15). For example, Okyar et al. (2012) found that the activity of $\mathrm{P}$-gp in the intestine is higher during the active period of rats (13). Future studies investigating the activity of P-gp in different cell types of the CNS across the 24-h cycle may provide further information on the molecular mechanisms underlying the findings presented in this study.

In addition to 24-h variation in $\mathrm{P}-\mathrm{gp}$ activity, the PBPK model (17) that was used to describe the effect of time of administration on the pharmacokinetics of quinidine in the brain shows that CSF flow is larger during the 
a

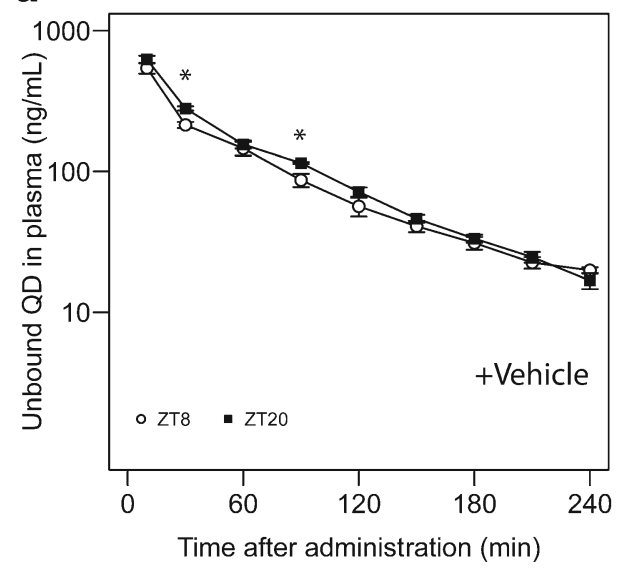

C

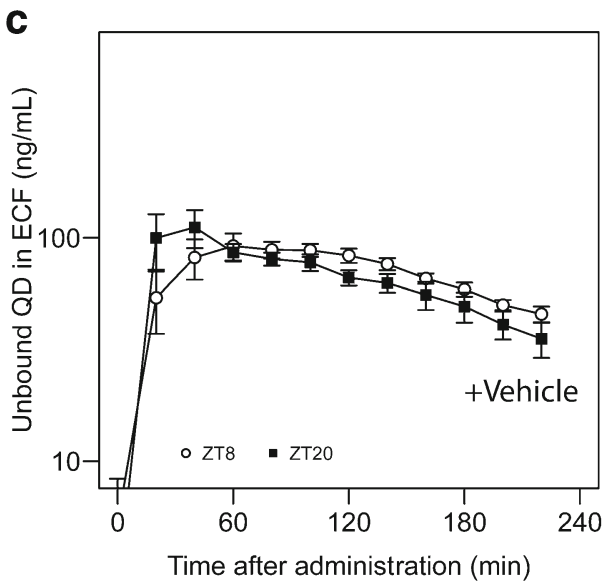

e

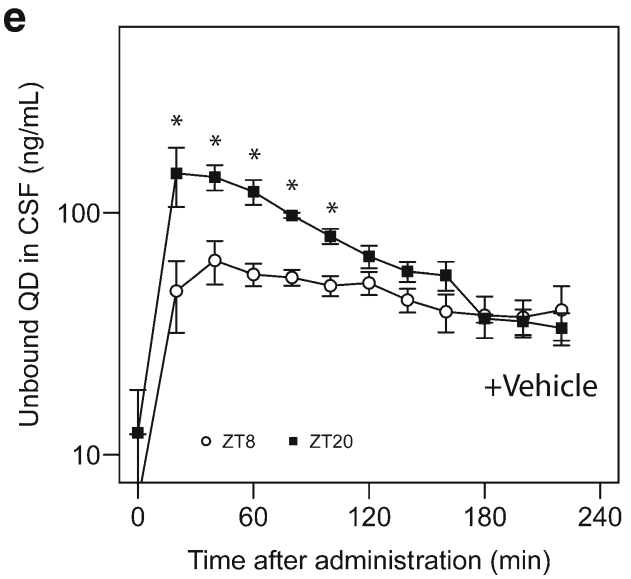

b
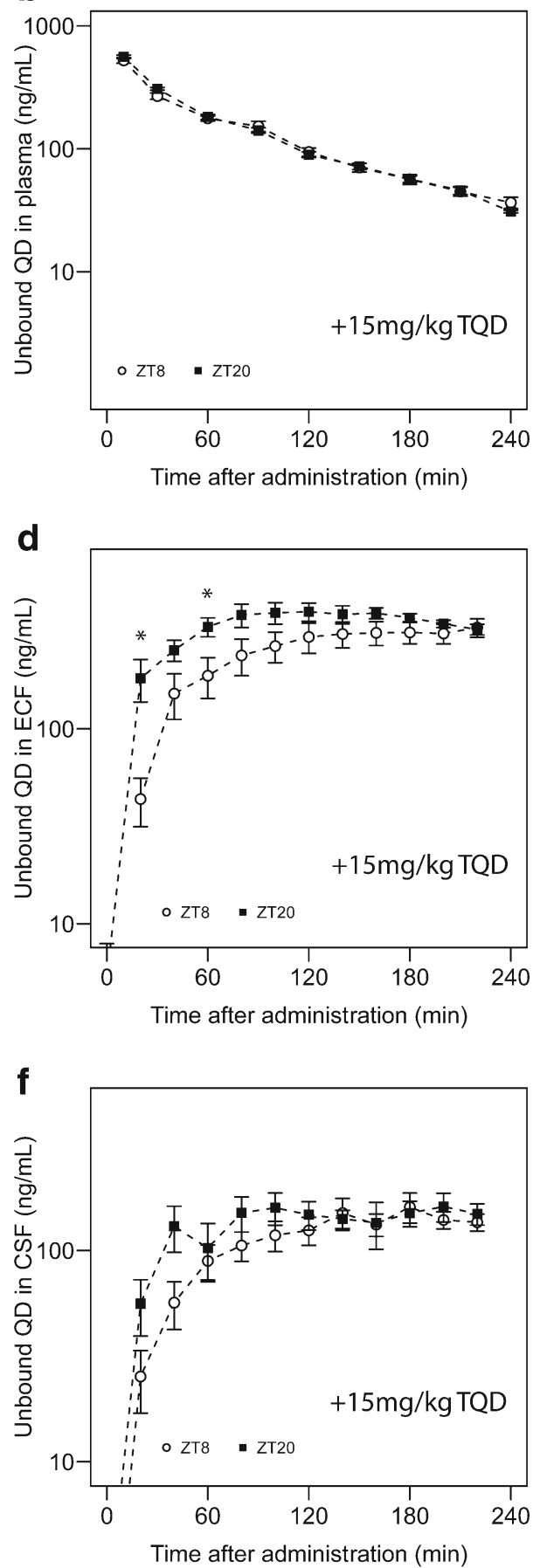

Fig. 3. Microdialysis experiments in vehicle- and TQD-treated animals. Time-concentration profiles of unbound quinidine in plasma (a, b, $n=7-8$ animals/group), ECF (c, d, $n=7-8$ animals/group), and CSF (e, $\mathbf{f}$, $n=6-8$ animals/group) at ZT8 (open symbols) and ZT20 (closed symbols) in vehicle-treated animals (a, c, e) and TQD-treated animals $(\mathbf{b}, \mathbf{d}, \mathbf{f})$. Asterisks indicate significant effect of ZT on quinidine concentration at the designated sampling times $(p<0.05$, two-sided $t$ test or Mann-Whitney $U$ test). Mean \pm SEM in all graphs. * $p<0.05$

resting period of the animals compared to the active period. In line with these findings, a recent study showed that sleep markedly increases CSF influx, thereby facilitating the removal of metabolic waste products from the CNS (22). This process may also affect the distribution of peripherally administered drugs in the brain. With the design used in this study, we cannot exclude the possibility that the sleep/wake state of the animal, rather than diurnal rhythmicity as such, is responsible for the variation in CSF flow or P-gp function over the 24-h period.

The variation in P-gp activity over the 24-h cycle had a much larger effect on quinidine concentrations in the brain 
Table I. Covariate Analysis

\begin{tabular}{lll}
\hline Covariate & Change in OFV & $p$ value \\
\hline Time of administration $(\mathrm{P}-\mathrm{gp})$ & -155.6 & $<0.05$ \\
Time of administration $\left(\mathrm{Q}_{\mathrm{CSF}}\right)$ & -37.7 & $<0.05$ \\
Time of administration $\left(\mathrm{P}-\mathrm{gp}\right.$ and $\left.\mathrm{Q}_{\mathrm{CSF}}\right)$ & -190.5 & $<0.05$ \\
\hline
\end{tabular}

${ }^{a}$ Change in objective function value (OFV) after addition of the covariate on various parameters compared to the model that does not take into account time of administration on any of the parameters

Table II. Parameter Estimates of the PBPK Model

\begin{tabular}{|c|c|c|c|c|}
\hline Parameter & & & Value & RSE (\%) \\
\hline \multicolumn{5}{|l|}{ Estimates } \\
\hline \multirow{2}{*}{$\mathrm{CL}_{\mathrm{E}}$} & Passive & & $83.3 \mathrm{~mL} / \mathrm{min}$ & 10.3 \\
\hline & +P-gp & & $52.7 \mathrm{~mL} / \mathrm{min}$ & 6.5 \\
\hline $\mathrm{Q}_{\text {PL-PER1 }}$ & Passive & & $831 \mathrm{~mL} / \mathrm{min}$ & 9.6 \\
\hline QPL-PER2 & Passive & & $93.5 \mathrm{~mL} / \mathrm{min}$ & 18.3 \\
\hline \multirow[t]{2}{*}{$\mathrm{CL}_{\text {PL-Deep Brain }}$} & Passive & & $982 \mu \mathrm{L} / \mathrm{min}$ & 14.7 \\
\hline & $+\mathrm{P}-\mathrm{gp}$ & & $\mathrm{NA}^{\mathrm{a}}$ & \\
\hline \multirow[t]{3}{*}{$\mathrm{CL}_{\text {Deep Brain-PL }}$} & Passive & & $12.3 \mu \mathrm{L} / \mathrm{min}$ & 19.8 \\
\hline & +P-gp & Resting period & $228 \mu \mathrm{L} / \mathrm{min}$ & 17.7 \\
\hline & $+\mathrm{P}-\mathrm{gp}$ & Active period & $659 \mu \mathrm{L} / \mathrm{min}$ & 15.6 \\
\hline \multirow[t]{3}{*}{$\mathrm{CL}_{\mathrm{PL-ECF}}$} & Passive & & $25.7 \mu \mathrm{L} / \mathrm{min}$ & 12.5 \\
\hline & $+\mathrm{P}-\mathrm{gp}$ & Resting period & $17 \mu \mathrm{L} / \mathrm{min}$ & 18.5 \\
\hline & $+\mathrm{P}-\mathrm{gp}$ & Active period & $18.3 \mu \mathrm{L} / \mathrm{min}$ & 18.5 \\
\hline \multirow[t]{3}{*}{$\mathrm{CL}_{\mathrm{ECF}-\mathrm{PL}}$} & Passive & & $4.63 \mu \mathrm{L} / \mathrm{min}$ & 15.2 \\
\hline & $+\mathrm{P}-\mathrm{gp}$ & Resting period & $3.14 \mu \mathrm{L} / \mathrm{min}$ & 36.3 \\
\hline & $+\mathrm{P}-\mathrm{gp}$ & Active period & $3.98 \mu \mathrm{L} / \mathrm{min}$ & 48.0 \\
\hline \multirow[t]{3}{*}{$\mathrm{CL}_{\mathrm{PL}-\mathrm{LV}}$} & Passive & & $3.23 \mu \mathrm{L} / \mathrm{min}$ & 19.9 \\
\hline & +P-gp & Resting period & $1.55 \mu \mathrm{L} / \mathrm{min}$ & 30.1 \\
\hline & $+\mathrm{P}-\mathrm{gp}$ & Active period & $2.44 \mu \mathrm{L} / \mathrm{min}$ & 17.5 \\
\hline \multirow[t]{2}{*}{$\mathrm{CL}_{\mathrm{LV}-\mathrm{PL}}$} & Passive & & $0.513 \mu \mathrm{L} / \mathrm{min}$ & 24.0 \\
\hline & $+\mathrm{P}-\mathrm{gp}$ & & $\mathrm{NA}^{\mathrm{a}}$ & \\
\hline \multirow[t]{2}{*}{$\mathrm{CL}_{\mathrm{PL}-\mathrm{CM}}$} & Passive & & $0.753 \mu \mathrm{L} / \mathrm{min}$ & 23.5 \\
\hline & $+\mathrm{P}-\mathrm{gp}$ & & $\mathrm{NA}^{\mathrm{a}}$ & \\
\hline \multirow[t]{2}{*}{$\mathrm{CL}_{\mathrm{CM}-\mathrm{PL}}$} & Passive & & $1.02 \mu \mathrm{L} / \mathrm{min}$ & 33.7 \\
\hline & $+\mathrm{P}-\mathrm{gp}$ & & $\mathrm{NA}^{\mathrm{a}}$ & \\
\hline $\mathrm{Q}_{\mathrm{ECF}}^{\mathrm{b}}$ & & & $0.2 \mu \mathrm{L} / \mathrm{min}$ & \\
\hline \multirow[t]{2}{*}{$\mathrm{Q}_{\mathrm{CSF}}$} & & Resting period & $0.522 \mu \mathrm{L} / \mathrm{min}$ & 28.5 \\
\hline & & Active period & $0.227 \mu \mathrm{L} / \mathrm{min}$ & 36.0 \\
\hline \multicolumn{2}{|l|}{$\mathrm{V}_{\mathrm{PL}}^{\mathrm{b}}$} & & $10.6 \mathrm{~mL}$ & \\
\hline \multicolumn{2}{|l|}{$\mathrm{V}_{\mathrm{PER}}{ }^{1}$} & & $7.42 \mathrm{~L}$ & 5.7 \\
\hline \multicolumn{2}{|l|}{$\mathrm{V}_{\mathrm{PER}}^{2}$} & & $7.09 \mathrm{~L}$ & 17.3 \\
\hline \multicolumn{2}{|l|}{$\mathrm{V}_{\text {Deep Brain }}{ }^{\mathrm{b}}$} & & $1440 \mu \mathrm{L}$ & \\
\hline \multicolumn{2}{|l|}{$\mathrm{V}_{\mathrm{ECF}}$} & & $290 \mu \mathrm{L}$ & \\
\hline \multicolumn{2}{|l|}{$V_{L V}{ }^{b}$} & & $50 \mu \mathrm{L}$ & \\
\hline \multicolumn{2}{|l|}{$\mathrm{V}_{\mathrm{TFV}}^{\mathrm{bV}}$} & & $50 \mu \mathrm{L}$ & \\
\hline \multicolumn{2}{|l|}{$\mathrm{V}_{\mathrm{CM}}$} & & $17 \mu \mathrm{L}$ & \\
\hline \multicolumn{2}{|l|}{$\begin{array}{l}v_{\mathrm{CM}}{ }^{b} \\
\mathrm{~V}_{\mathrm{SAS}}\end{array}$} & & $180 \mu \mathrm{L}$ & \\
\hline \multicolumn{5}{|l|}{ Inter-individual variability } \\
\hline$C L_{E}$ & & & $33.2 \%$ & 17.2 \\
\hline \multicolumn{5}{|l|}{ Residual error } \\
\hline PL & & & $42.8 \%$ & 13.9 \\
\hline $\mathrm{ECF}$ & & & $33.0 \%$ & 13.9 \\
\hline LV & & & $31.9 \%$ & 18.7 \\
\hline $\mathrm{CM}$ & & & $36.2 \%$ & 13.8 \\
\hline Deep brain & & & $35.6 \%$ & 13.4 \\
\hline Objective function value & & & $30,298.06$ & \\
\hline
\end{tabular}

PER1 peripheral compartment 1, PER2 peripheral compartment 2, $P L$ plasma, $E C F$ brain extracellular fluid, $L V$ lateral ventricle, $C M$ cisterna magna, $Q E C F$ ECF flow, $Q C S F$ CSF flow, $V$ volume of distribution of the compartments, $T F V$ third and fourth ventricle, $S A S$ subarachnoid space, $+P$ - $g p$ effect of P-gp-mediated transport on the parameter

${ }^{a}$ Parameter not available

${ }^{b}$ Physiological values derived from literature (17) 
tissue than in ECF. Total brain concentrations reflect the concentration of quinidine in both ECF and intracellular fluid (ICF) of brain parenchymal cells. The relatively small difference in the effect of P-gp on the clearance of quinidine from ECF to plasma during the active and resting periods of the animals cannot account for the large difference in the effect of P-gp on the clearance of quinidine from brain tissue to plasma. Therefore, we propose that an additional P-gpdependent barrier between the ECF or plasma and the ICF of brain parenchymal cells gives rise to the observed variation. The existence of an additional barrier that affects the distribution of drugs in parenchymal cells has previously been suggested by the observation that valproic acid, an anticonvulsant drug, is subject to active efflux at the parenchymal cell membrane (23). Although valproic acid is not a P-gp substrate (24), Scism et al. (23) show that the parenchymal cell membrane can play an important role in the distribution of drugs in the brain. Furthermore, Syvänen et al. (19) show that induction of status epilepticus in rats affects Pgp activity at the level of the parenchyma rather than at the BBB.

Several lines of evidence suggest that P-gp is present in brain parenchymal cells, such as in pericytes and astrocytes $(4,25,26)$. P-gp does not seem to be present in neurons in the healthy mammalian brain, but its expression is rapidly induced when challenged, for example, by hypoxic stress or the induction of status epilepticus $(27,28)$. Importantly, it should be kept in mind that experiments are generally conducted during the resting period of the animals, which is the time window during which $\mathrm{P}$-gp activity is lowest according to our findings. The existence of a barrier from the ECF to the ICF is of special interest for drugs that have an intracellular CNS target. Future studies should aim to elucidate this process more clearly.

A question that needs to be addressed is to what extent a twofold increase in total brain exposure is clinically relevant. Several strategies have been proposed to minimize the effect of P-gp on its substrates that are used as drugs to treat various CNS disorders. For example, pharmacological inhibition of Pgp by tariquidar in humans leads to a dose-dependent increase in brain uptake of a P-gp substrate $(29,30)$. At a dose of $6 \mathrm{mg} / \mathrm{kg}$, there is a fourfold increase in brain uptake of radiolabeled loperamide (29). However, this dose requires intravenous infusion for more than $1 \mathrm{~h}$ due to a hemolytic effect of one the co-solvents $(29,31)$. Another limitation associated with P-gp inhibitors in humans is that the unbound plasma concentrations are relatively low, which limits the degree of P-gp inhibition (32). In light of these findings, the more than twofold increase in brain exposure that was observed in this study, by investigating a naturally occurring physiological process, could therefore well be of clinical relevance. This holds especially true for drugs with a narrow therapeutic index in which a slight increase in brain concentration leads to a large increase in effect size.

In summary, this study indicates that the exposure to a Pgp substrate in the brain is subject to diurnal variation. Using a selective and potent P-gp inhibitor, we are able to show that this is due to variation of P-gp-mediated transport which is markedly elevated during the active period of the animals compared to the resting period. Furthermore, CSF flux is increased during the resting period, which may also cause variations in the exposure to a drug in the brain. Importantly, these findings emphasize the need to take into account the timing of drug administration, both in clinical and experimental situations. Dosing at the appropriate time of the day may be an effective strategy to modulate the delivery of P-gp substrates to the brain.

Conflict of interest The authors declare no conflict of interest.

Funding This research was supported by the Dutch Technology Foundation STW, which is the applied science division of NWO, and the Technology Programme of the Ministry of Economic Affairs.

Open Access This article is distributed under the terms of the Creative Commons Attribution License which permits any use, distribution, and reproduction in any medium, provided the original author(s) and the source are credited.

\section{REFERENCES}

1. Abbott NJ, Patabendige AAK, Dolman DEM, Yusof SR, Begley DJ. Structure and function of the blood-brain barrier. Neurobiol Dis. 2010;37:13-25.

2. Ohtsuki S, Terasaki T. Contribution of carrier-mediated transport systems to the blood-brain barrier as a supporting and protecting interface for the brain; importance for CNS drug discovery and development. Pharm Res. 2007;24:1745-58.

3. Schinkel AH, Smit JJ, van Tellingen O, Beijnen JH, Wagenaar E, van Deemter L, et al. Disruption of the mouse mdr1a Pglycoprotein gene leads to a deficiency in the blood-brain barrier and to increased sensitivity to drugs. Cell. 1994;77:491-502.

4. Bendayan R, Ronaldson PT, Gingras D, Bendayan M. In situ localization of P-glycoprotein (ABCB1) in human and rat brain. J Histochem Cytochem. 2006;54:1159-67.

5. Potschka H. Targeting regulation of ABC efflux transporters in brain diseases: a novel therapeutic approach. Pharmacol Ther. 2010;125:118-27.

6. Dallmann R, Brown SA, Gachon F. Chronopharmacology: new insights and therapeutic implications. Annu Rev Pharmacol Toxicol Ann Rev. 2014;54:339-61.

7. Watanabe J, Suzuki Y, Fukui N, Ono S, Sugai T, Tsuneyama N, et al. Increased risk of antipsychotic-related QT prolongation during nighttime: a 24-hour holter electrocardiogram recording study. J Clin Psychopharmacol. 2012;32:18-22.

8. Hermida RC, Ayala DE, Fernández JR, Calvo C. Chronotherapy improves blood pressure control and reverts the nondipper pattern in patients with resistant hypertension. Hypertension. 2008;51:69-76.

9. Buttgereit F, Doering G, Schaeffler A, Witte S, Sierakowski S, Gromnica-Ihle E, et al. Targeting pathophysiological rhythms: prednisone chronotherapy shows sustained efficacy in rheumatoid arthritis. Ann Rheum Dis. 2010;69:1275-80.

10. Ando H, Yanagihara H, Sugimoto K, Hayashi Y, Tsuruoka S, Takamura T, et al. Daily rhythms of P-glycoprotein expression in mice. Chronobiol Int. 2005;22:655-65.

11. Murakami Y, Higashi Y, Matsunaga N, Koyanagi S, Ohdo S. Circadian clock-controlled intestinal expression of the multidrugresistance gene mdr1a in mice. Gastroenterology. 2008;135:163644.e3.

12. Hayashi $\mathrm{Y}$, Ushijima $\mathrm{K}$, Ando $\mathrm{H}$, Yanagihara H, Ishikawa E, Tsuruoka S-I, et al. Influence of a time-restricted feeding schedule on the daily rhythm of abcb1a gene expression and its function in rat intestine. J Pharmacol Exp Ther. 2010;335:418-23.

13. Okyar A, Dressler C, Hanafy A, Baktir G, Lemmer B, SpahnLangguth $\mathrm{H}$. Circadian variations in exsorptive transport: in situ 
intestinal perfusion data and in vivo relevance. Chronobiol Int. 2012;29:443-53.

14. Ballesta A, Dulong S, Abbara C, Cohen B, Okyar A, Clairambault J, et al. A combined experimental and mathematical approach for molecular-based optimization of irinotecan circadian delivery. PLoS Comput Biol. 2011;7:e1002143.

15. Stearns AT, Balakrishnan A, Rhoads DB, Ashley SW, Tavakkolizadeh A. Diurnal rhythmicity in the transcription of jejunal drug transporters. J Pharmacol Sci. 2008;108:144-8.

16. De Lange ECM. The mastermind approach to CNS drug therapy: translational prediction of human brain distribution, target site kinetics, and therapeutic effects. Fluids Barriers CNS. 2013;10:12.

17. Westerhout J, Smeets J, Danhof M, de Lange ECM. The impact of P-gp functionality on non-steady state relationships between CSF and brain extracellular fluid. J Pharmacokinet Pharmacodyn. 2013;40:327-42.

18. Moghaddam B, Bunney BS. Ionic composition of microdialysis perfusing solution alters the pharmacological responsiveness and basal outflow of striatal dopamine. J Neurochem. 1989;53:652-4.

19. Syvänen S, Schenke M, van den Berg D-J, Voskuyl RA, de Lange EC. Alteration in P-glycoprotein functionality affects intrabrain distribution of quinidine more than brain entry-a study in rats subjected to status epilepticus by kainate. AAPS J. 2012;14:87-96.

20. Meijer JH, Colwell CS, Rohling JHT, Houben T, Michel S. Dynamic neuronal network organization of the circadian clock and possible deterioration in disease. Prog Brain Res. 2012;199:143-62.

21. Mohawk JA, Green CB, Takahashi JS. Central and peripheral circadian clocks in mammals. Annu Rev Neurosci Annu Rev. 2012;35:445-62.

22. Xie L, Kang H, Xu Q, Chen MJ, Liao Y, Thiyagarajan M, et al. Sleep drives metabolite clearance from the adult brain. Science. 2013;342:373-7.

23. Scism JL, Powers KM, Artru AA, Lewis L, Shen DD. Probenecid-inhibitable efflux transport of valproic acid in the brain parenchymal cells of rabbits: a microdialysis study. Brain Res. 2000;884:77-86.

24. Baltes S, Fedrowitz M, Tortós CL, Potschka H, Löscher W. Valproic acid is not a substrate for P-glycoprotein or multidrug resistance proteins 1 and 2 in a number of in vitro and in vivo transport assays. J Pharmacol Exp Ther. 2007;320:331-43.

25. Golden PL, Pardridge WM. P-glycoprotein on astrocyte foot processes of unfixed isolated human brain capillaries. Brain Res. 1999;819:143-6.

26. Shimizu F, Sano Y, Maeda T, Abe M-A, Nakayama H, Takahashi R-I, et al. Peripheral nerve pericytes originating from the bloodnerve barrier expresses tight junctional molecules and transporters as barrier-forming cells. J Cell Physiol. 2008;217:388-99.

27. Volk H, Burkhardt K, Potschka H, Chen J, Becker A, Löscher W. Neuronal expression of the drug efflux transporter Pglycoprotein in the rat hippocampus after limbic seizures. Neuroscience. 2004;123:751-9.

28. Lazarowski A, Caltana L, Merelli A, Rubio MD, Ramos AJ, Brusco A. Neuronal mdr-1 gene expression after experimental focal hypoxia: a new obstacle for neuroprotection? J Neurol Sci. 2007;258:84-92.

29. Kreisl WC, Liow J-S, Kimura N, Seneca N, Zoghbi SS, Morse $\mathrm{CL}$, et al. P-glycoprotein function at the blood-brain barrier in humans can be quantified with the substrate radiotracer $11 \mathrm{C}-\mathrm{N}$ desmethyl-loperamide. J Nucl Med. 2010;51:559-66.

30. Wagner CC, Bauer M, Karch R, Feurstein T, Kopp S, Chiba P, et al. A pilot study to assess the efficacy of tariquidar to inhibit Pglycoprotein at the human blood-brain barrier with (R)-11Cverapamil and PET. J Nucl Med. 2009;50:1954-61.

31. Bauer M, Zeitlinger M, Todorut D, Böhmdorfer M, Müller M, Langer $\mathrm{O}$, et al. Pharmacokinetics of single ascending doses of the P-glycoprotein inhibitor tariquidar in healthy subjects. Pharmacology. 2013;91:12-9.

32. Kalvass JC, Polli JW, Bourdet DL, Feng B, Huang S-M, Liu X, et al. Why clinical modulation of efflux transport at the human blood-brain barrier is unlikely: the ITC evidence-based position. Clin Pharmacol Ther. 2013;94:80-94. 